\title{
FITOEXTRAÇÃO DE CÁDMIO E ZINCO DE UM LATOSSOLO VERMELHO-AMARELO CONTAMINADO COM METAIS PESADOS $\left({ }^{\mathbf{1}}\right)$
}

\author{
CAROLINA DE FREITAS ZEITOUNI $\left({ }^{2}\right)$; RONALDO SEVERIANO BERTON $\left({ }^{2}\right)$; \\ CLEIDE APARECIDA DE ABREU $(2,3)$
}

\begin{abstract}
RESUMO
Avaliou-se a capacidade das plantas de mamona, girassol, pimenta da amazônia e tabaco, cultivadas em vasos de $3 \mathrm{~L}$, em casa de vegetação, em extrair metais de um Latossolo Vermelho-Amarelo distrófico. O delineamento experimental foi em blocos ao acaso, esquema fatorial 4 espécies $\times 3$ doses de metais $\times 2$ doses de EDTA (Ácido Etilenodiaminotetraacético), com três repetições. As doses empregadas de metais, aplicadas na forma de sais inorgânicos foram, em $\mathrm{mg} \mathrm{kg}^{-1}$ : dose zero - sem adição de metal; dose 1 adição conjunta de 0,75 de $\mathrm{Cd}$, 6,25 de $\mathrm{Pb}, 20,0$ de $\mathrm{Cu}, 8,75$ de Ni e 62,5 de Zn e; dose 2 - adição conjunta de 1,5 de $\mathrm{Cd}, 12,5$ de $\mathrm{Pb}, 40,0$ de $\mathrm{Cu}, 17,5$ de $\mathrm{Ni}$ e 125,0 de $\mathrm{Zn}$, na presença $\left(1 \mathrm{mmol} \mathrm{kg}^{-1}\right)$ e ausência de EDTA. A colheita da parte aérea das quatro espécies de plantas variou de 48 dias (pimenta) até 100 dias (tabaco). A aplicação do EDTA diminuiu a produção de matéria seca da parte aérea de todas as plantas, embora tenha provocado aumento na concentração de $\mathrm{Cd}$ na parte aérea da mamoneira e do girassol e nenhuma influência na concentração de Cd (pimenta e tabaco) e na de $\mathrm{Zn}$ em todas as espécies. Concluiuse que a planta mais eficiente em extrair o Cd e o Zn do solo enriquecido com metais foi o tabaco (Nicotiana tabacum) e que para descontaminar o solo, será necessário um maior número de cultivos para todas as culturas, com exceção da mamoneira, se houver a aplicação de EDTA na dose de $1 \mathrm{mmol} \mathrm{kg}^{-1}$.
\end{abstract}

Palavras-chave: fitorremediação, EDTA, tabaco, mamona, pimenta, girassol, poluição do solo.

\section{ABSTRACT \\ PHYTOEXTRACTION OF CADMIUM AND ZINC FROM AN OXISOL CONTAMINATED WITH HEAVY METALS}

In a greenhouse experiment, the ability of sunflower (Helianthus annuus L.), castor bean (Ricinus communis L.), tobacco (Nicotiana tabacum L.), and Amazon pepper (Capsicum baccatum L.) to extract Cd and $\mathrm{Zn}$ from an oxisol contaminated with heavy metals was evaluated. The experiment was carried out in a complete ramdomized block design in a factorial scheme consisted of 4 species $\times 3$ rates of metals $\times 2$ rates of EDTA, with 3 replicates and using plastic pots with $3 \mathrm{~L}$ capacity. Heavy metals were applied to the soil, as inorganic salts, at rates, in $\mathrm{mg} \mathrm{kg}^{-1}$ : dose 0 : control (no metals added); dose 1: - combined application of 0.75 of $\mathrm{Cd} ; 6.25$ of $\mathrm{Pb} ; 20.0$ of $\mathrm{Cu} ; 8.75$ of $\mathrm{Ni}$ and 62.5 of $\mathrm{Zn}$; and dose 2 - combined application of 1.5 of $\mathrm{Cd} ; 12.5$ of $\mathrm{Pb} ; 40.0$ of $\mathrm{Cu} ; 17.5$ of $\mathrm{Ni}$ and 125,0 of $\mathrm{Zn}$, with and without EDTA addition of 1 $\mathrm{mmol} \mathrm{kg} \mathrm{k}^{-1}$. Plant tops were harvested from 48 to 100 days after sowing or seedlings transplant to the pots. EDTA addition decreased dry matter yield of all species, although it contributed to the increase of shoot $\mathrm{Cd}$ concentration on castor bean and sunflower plants, and had no effect on $\mathrm{Zn}$ shoot concentration for all species under investigation. Tobbaco was the most efficient specie in extracting $\mathrm{Zn}$ and $\mathrm{Cd}$ from the contaminated soil. A great number of sunflower, Amazon pepper and tobacco cropping will be necessary for soil decontamination, if it receives the addition of EDTA at rate of $1 \mathrm{mmol} \mathrm{kg}{ }^{-1}$.

Key words: Phytoremediation, EDTA, tobacco, castor bean, pepper, sunflower, soil pollution.

$\left({ }^{1}\right)$ Recebido para publicação em 17 de agosto de 2006 e aceito em

$\left({ }^{2}\right)$ Centro de Pesquisa e Desenvolvimento de Solos e Recursos Ambientais, Instituto Agronômico, Av. Barão de Itapura, 1481, Caixa Postal 28, 13020-902 Campinas (SP).E-mail: rsberton@iac.sp.gov.br $\left(^{*}\right)$ Autor correspondente; cleide@iac.sp.gov.br; carolfz@pop.com.br

$\left({ }^{3}\right)$ Bolsista do CNPq. 


\section{INTRODUÇÃO}

O aumento das atividades industriais e de mineração aliado ao uso inadequado de fertilizantes e pesticidas no solo têm contribuído para a contaminação do solo, cursos de água e lençol freático por metais pesados.

Uma determinada área é considerada contaminada se, entre outros fatores, as concentrações de elementos ou substâncias de interesse ambiental estão acima de um dado limite denominado valor de intervenção. Acima desse limite, há um risco potencial de efeito deletério sobre a saúde humana, havendo necessidade de uma ação imediata na área. Até novembro de 2006, o cadastro de locais contaminados no Estado de São Paulo apontou a existência de 1.822 locais, sendo 238 com metais pesados (CЕTESB, 2007).

A busca e o desenvolvimento de soluções tecnológicas que atendam à legislação ambiental se fazem primordial. Neste contexto, destaca-se a fitoextração, que utiliza plantas para remover os metais do solo acumulando-os nas raízes e parte aérea. É uma tecnologia de baixo custo (TANDY et al., 2004) e o seu sucesso depende, do grau de contaminação do metal no solo, da capacidade das plantas em acumularem o metal na parte aérea e da disponibilidade do metal no solo (ERNEST, 1996). Essa técnica possui outros benefícios como a manutenção da fertilidade do solo e a grande aceitação pública por ser uma tecnologia 'verde', além de ter a luz solar como principal fonte de energia (Usepa 2004; Pereira, 2005).

As plantas hiperacumuladoras são altamente especializadas em acumular ou tolerar altíssimas concentrações elevadas de metais como: $>10.000 \mathrm{mg}$ $\mathrm{kg}^{-1}$ de $\mathrm{Zn}$ e $\mathrm{Mn}$; $>1.000 \mathrm{mg} \mathrm{kg}^{-1}$ de $\mathrm{Pb}$, $\mathrm{Ni}$ e $\mathrm{Cu}$; > $100 \mathrm{mg} \mathrm{kg}^{-1}$ de Cd (RASKIN et al., 1994). Até o momento, foram identificadas 400 plantas hiperacumuladoras, sendo a maioria originária de áreas contaminadas da Europa, Estados Unidos, Nova Zelândia e Austrália (KHAN et al., 2000). Essas plantas pertencem às famílias Brassicaceae, Fabaceae, Euphorbiaceae, Asteraceae, Lamiaceae e Scrophulariaceae (GARbisu e AlKorta, 2001). Algumas plantas possuem potencial para extrair vários metais do solo, outras são mais específicas. A Brassica juncea possui potencial para remediar solos com altos teores de $\mathrm{Pb}, \mathrm{Cr}, \mathrm{Cd}, \mathrm{Cu}, \mathrm{Ni}$, $\mathrm{Zn}, \mathrm{Sr}, \mathrm{B}$ e Se; Thlaspi caerulescens para fitorremediar $\mathrm{Cd}$, Ni e Zn; Helianthus annuus, Nicotiana tabacum e Alyssum wufenianum para extrair Ni (UsePa, 2000).

Os mecanismos envolvidos na tolerância das plantas a altas concentrações de metais no solo são vários e, ainda, não muito bem definidos. Estes estão relacionados às diferenças na estrutura e no funcionamento das membranas celulares, na remoção de íons do metabolismo por armazenamento em formas fixas e/ou insolúveis em vários órgãos e organelas, alteração em padrões metabólicos, dentre outros (MoHr e SCHOPfer, 1995). De acordo com esses autores, a formação de fitoquelatinas foi a principal razão para a tolerância de algumas espécies aos altos teores de $\mathrm{Zn}$ e $\mathrm{Cd}$ no solo.

O aumento da concentração de metais pesados no citoplasma das plantas leva à ativação da síntese de fitoquelatinas, que seqüestra os íons metálicos, evitando concentrações críticas desses nas células. Estudos de tolerância ao $\mathrm{Pb}$, realizados com algumas variedades das espécies de Vigna e Orysa, demonstraram que as variedades mais tolerantes tinham maior atividade de peroxidase, catalase e glucose-6-fosfato dehidrogenase em relação àquelas não tolerantes (Routh et al., 2001). AsHOMORE (2000) comenta que as plantas tolerantes cultivadas ou presentes em áreas contaminadas por metais pesados desenvolvem barreiras reprodutivas, como o florescimento precoce.

Uma das limitações da fitoextração para remediar áreas contaminadas por metais pesados devese à baixa disponibilidade desses elementos no solo. Alguns autores têm sugerido a utilização de agentes quelantes que, segundo SHEN et al. (2002), podem dessorver o metal da matriz do solo para formar complexo solúvel, na solução do solo. A formação do complexo metálico é muito estável e, normalmente, não libera o íon metálico para a forma livre, a menos que haja uma queda significativa no $\mathrm{pH}$ do solo (KHAN et al., 2000). Por isso, a formação do complexo quelato/ metal previne a precipitação e a sorção do metal e mantém sua disponibilidade para as plantas (GARBISU e Alcorta, 2001).

Recentemente, agentes quelantes de baixa toxidez, como o NTA (ácido nitrilotriacético) e o EDTA (ácido etilenodiaminotetraacético), vêm sendo utilizados para aumentar a biodisponibilidade de metais pesados para as plantas. De acordo com TANDY et al. (2004), o quelante EDTA pode aumentar de $45 \%$ a $100 \%$ a eficiência de remoção do $\mathrm{Pb}$ de solos contaminados. Resultados observados por cientistas em outros países mostraram grande eficiência do EDTA no aumento da concentração de $\mathrm{Pb}$ na parte aérea de diversas culturas. HuANG et al. (1997), trabalhando com solo contaminado por $\mathrm{Pb}(2500 \mathrm{mg}$ $\left.\mathrm{kg}^{-1}\right)$, obtiveram um aumento de 100 para $3.000 \mathrm{mg}$ $\mathrm{kg}^{-1}$ de $\mathrm{Pb}$ em diferentes espécies. KHAN et al. (2000) verificaram que, quando foi aplicada a dose de $1 \mathrm{~g}$ $\mathrm{kg}^{-1}$ de solo de EDTA ou DTPA (dietileno triaminopentaacético) em solos contaminados com metais pesados, houve aumento na absorção de $\mathrm{Fe}, \mathrm{Mn}$ 
e $\mathrm{Cu}$ pelas plantas de milho, cultivadas em casa de vegetação. Pereira (2005) verificou que, para o feijão de porco e girassol, a aplicação de $0,5 \mathrm{~g} \mathrm{~kg}^{-1}$ de EDTA foi eficiente em aumentar a absorção de $\mathrm{Pb}$, não o sendo para o milho. Esse autor observou que na presença do EDTA, mais de $90 \%$ do total das formas $\mathrm{de} \mathrm{Pb}, \mathrm{Fe}$ e $\mathrm{Al}$ presentes no solo foram complexados com o EDTA.

O estudo e a exploração comercial de plantas fitoextratoras estão bastante avançados nos países desenvolvidos e com políticas ambientais bem definidas. No Brasil, porém, essa técnica é pouco explorada por falta de capacitação técnica, desconhecimento do mercado e pelo fato de as plantas hiperacumuladoras conhecidas serem, em sua maioria, de clima temperado (PEREIRA, 2005). Portanto, o objetivo deste trabalho foi avaliar a capacidade extratora de metais pesados, principalmente $\mathrm{Cd}$ e $\mathrm{Zn}$, por mamona, girassol, pimenta da amazônia e tabaco, cultivadas em um Latossolo Vermelho-Amarelo Distrófico contaminado com metais pesados, na presença e ausência de EDTA.

\section{MATERIAL E MÉTODOS}

O experimento foi desenvolvido em casa de vegetação, em Campinas, utilizando-se amostra de um Latossolo Vermelho-Amarelo distrófico, da camada de 0-20 $\mathrm{cm}$ de profundidade, que recebeu calcário para elevar o $\mathrm{pH}$ em $\mathrm{CaCl}_{2}$ a 6,2 .

O delineamento experimental foi em blocos ao acaso, esquema fatorial $4 \times 3 \times 2$, com três repetições.

Os tratamentos foram constituídos de quatro espécies vegetais: girassol (Helianthus annus L.), mamoneira (Ricinus communis L.), tabaco (Nicotiana tabacum L.) e pimenta da Amazônia (Capsicum baccatum L.); três doses de metais pesados $(\mathrm{Cd}, \mathrm{Pb}, \mathrm{Cu}, \mathrm{Ni}$ e $\mathrm{Zn})$, e ausência e presença de $1 \mathrm{mmol} \mathrm{kg}{ }^{-1}$ de EDTA $(0,372$ $\mathrm{mg} \mathrm{kg}^{-1}$ ), conforme descrito adiante. As doses de metais foram proporcionais às concentrações de metais pesados constatadas no lodo de esgoto de Barueri (SP). O cálculo foi feito com base na concentração de $\mathrm{Zn}$ presente no lodo de esgoto, considerando a adição no solo de $0,62,5$ e $125 \mathrm{mg} \mathrm{dm}^{-3}$ de Zn. Portanto, no experimento foram utilizadas as seguintes doses, em mg kg-1 de: dose zero - sem adição de metal; dose 1 - adição conjunta de 0,75 de Cd, 6,25 de $\mathrm{Pb}, 20,0$ de $\mathrm{Cu}, 8,75$ de Ni e 62,5 de $\mathrm{Zn}$, e dose 2 adição conjunta de 1,5 de $\mathrm{Cd}, 12,5$ de $\mathrm{Pb}, 40,0$ de $\mathrm{Cu}$, 17,5 de Ni e 125,0 de Zn.

Os metais foram aplicados na forma de sais inorgânicos puros para análise usando-se como fontes o cloreto de cádmio, nitrato de chumbo, sulfato de cobre, sulfato de níquel e sulfato de zinco que foram misturados e homogeneizados ao material de solo contido em vasos de $3 \mathrm{~L}$, conforme o tratamento. Nesse momento e de maneira similar à aplicação dos metais, foram adicionados, em todos os tratamentos, o sulfato de amônio $\left(35,7 \mathrm{mg} \mathrm{dm}^{-3}\right.$ de $\left.\mathrm{N}\right)$, superfosfato simples $\left(142,86 \mathrm{mg} \mathrm{dm}^{-3}\right.$ de $\left.\mathrm{P}\right)$, cloreto de potássio $(100 \mathrm{mg}$ $\mathrm{dm}^{-3}$ de K) e ácido bórico $\left(0,75 \mathrm{mg} \mathrm{dm}^{-3} \mathrm{de} \mathrm{B}\right)$.

Após a adição dos metais e da adubação básica, as amostras de solo permaneceram incubadas por 25 dias, mantendo-se o teor de umidade a $50 \%$ de sua capacidade máxima de retenção de água, por meio de pesagem diária dos vasos, aplicando-se água destilada quando necessário.

Logo após a incubação com os metais pesados, retirou-se uma amostra de terra de cada vaso para determinação dos teores de $\mathrm{Cd}, \mathrm{Cu}, \mathrm{Ni}, \mathrm{Pb}$ e $\mathrm{Zn}$ em ácido nítrico, usando o método SW 846-3051, conforme descrito por Abreu et al. (2001).

Em seguida, fez-se a semeadura do girassol e da mamona, deixando-se uma planta por vaso, após o desbaste. No caso do tabaco e da pimenta, foi transplantada uma muda por vaso, previamente cultivada por 117 dias em substrato (mistura de casca de pinus e carvão.

O agente quelatizante EDTA, na concentração de $0,372 \mathrm{mg} \mathrm{kg}^{-1}$ de solo, foi aplicado em forma de solução na superfície do solo, 44 dias após a germinação do girassol e da mamona e 53 dias após o transplante das mudas de tabaco e pimenta da amazônia, nos respectivos tratamentos.

Durante o período do experimento, foram feitas aplicações de cobertura de nitrogênio, cujo intervalo entre elas dependeu da exigência da cultura, totalizando, em mg dm${ }^{-3}: 476$ (tabaco), 214 (mamona), 238 (pimenta) e 214 (girassol). O teor de umidade das amostras de solo foi mantido a $60 \%$ da capacidade máxima de retenção de água, por meio de pesagem diária dos vasos, e adição de água destilada de acordo com a necessidade.

As partes aéreas das culturas foram colhidas em dias diferentes em função da capacidade de sobrevivência das plantas em relação às condições adversas dos tratamentos. Para as culturas da mamona e tabaco, a colheita foi aos 90 e 100 dias após a germinação ou transplante, respectivamente. A parte aérea da pimenta foi colhida aos 57 dias após o transplante para os tratamentos que receberam EDTA e aos 98 dias para aqueles que não receberam. A colheita da parte aérea do girassol foi aos 48 dias após a germinação para todos os tratamentos que receberam a dose 2 de metais; aos 64 dias para a dose zero e dose 1 com EDTA, e aos 76 dias para a dose zero e dose 1 sem EDTA. 
As plantas foram cortadas a $1 \mathrm{~cm}$ da superfície do solo e lavadas em uma solução de detergente $(0,1 \% \mathrm{v} / \mathrm{v})$, enxaguadas em água corrente e deionizada, secas em estufa com circulação forçada de ar à temperatura entre 65 e $70{ }^{\circ} \mathrm{C}$, até massa constante. Em seguida, as amostras foram pesadas, moídas em moinho tipo Wiley, e submetidas à digestão nitroperclórica (BATAGLIA et al., 1983), para determinação dos metais pesados por espectrometria de emissão atômica com plasma.

Os dados foram submetidos à análise de variância, utilizando o Teste de Tukey, a 5\% de probabilidade, para interpretar as diferenças significativas entre as médias. O programa computacional utilizado para a análise estatística foi o SANEST (ZONTA et al., 1984).

Fez-se o balanço de massa usando os seguintes cálculos: a) quantidade de $\mathrm{Cd}$ e $\mathrm{Zn}$ a ser removido do solo $=$ teor total de $\mathrm{Zn}$ ou $\mathrm{Cd}$ no solo após a aplicação de metais no solo $\left(\mathrm{mg} \mathrm{kg}^{-1}\right)$ - o teor total desses antes da aplicação $\left(\mathrm{mg} \mathrm{kg}^{-1}\right)$; b) teor de metal a ser removido por vaso $=\mathrm{o}$ teor de metal a ser removido do solo $\left(\mathrm{mg}^{\mathrm{kg}}{ }^{-1}\right) \times 3 \mathrm{~kg}$ de solo; c) remoção de metal $(\%)=$ teor acumulado de metal (mg por vaso) x 100 / teor a ser removido do metal por vaso (mg por vaso) c) número de cultivos necessários para descontaminar a amostra de solo do vaso $=$ teor de metal a ser removido da terra de cada vaso / teor de metal acumulado na parte aérea de cada planta; d) matéria seca total produzida = matéria seca produzida $\mathrm{x}$ número de cultivos necessários para descontaminar a área.

\section{RESULTADOS E DISCUSSÃO}

\subsection{Sintomas visuais}

Na mamoneira, observou-se amarelecimento das folhas e crescimento lento logo aos dois dias após a germinação, nos tratamentos que receberam a maior dose de metais, independentemente da adição ou não do quelante. Nos tratamentos que receberam o quelante os sintomas se agravaram para manchas pretas nas folhas mais velhas, e conseqüente queda.

Os sintomas visuais semelhantes àqueles descritos para a mamona foram observados para o girassol e a pimenta. Contudo, após a aplicação do EDTA, as manchas pretas nas folhas mais velhas do girassol e pimenta estavam mais evidentes, levando à paralisação do crescimento. Esses sintomas não foram observados na ausência de EDTA.

Na cultura do tabaco, foram observados sintomas visuais de toxicidade após dez dias da aplicação do EDTA, que correspondeu 52 dias após o transplante das mudas, quando nas folhas mais velhas ocorreram manchas castanhas e começaram a secar. Com o passar do tempo o desenvolvimento das plantas foi prejudicado.

\subsection{Produção de matéria seca}

Considerando a produção de matéria seca da parte aérea de cada uma das espécies testadas, a aplicação de EDTA foi prejudicial, independentemente da dose de metal aplicada (Tabela 1). O efeito da aplicação do EDTA foi muito semelhante para todas as espécies, ou seja, causou uma redução entre $40 \%$ e $50 \%$ na produção de matéria seca, exceto para a dose 2 , cuja redução foi menor pela sua adição.

O efeito negativo do EDTA na produção de matéria seca das plantas esta relacionado com a absorção dos metais, que será discutido posteriormente. Menor produção de massa seca de espécies arbóreas, devido à aplicação do complexante EDTA em solo contaminado por metais, também foi verificada por MARQUES et al. (2000). Resultados semelhantes foram observados por CHEN e Cutright (2001), adicionando $0,5 \mathrm{~g} . \mathrm{kg}^{-1}$ de EDTA em solos contaminados por $\mathrm{Cd}, \mathrm{Cr}$ e $\mathrm{Ni}$.

$\mathrm{Na}$ ausência de EDTA, a produção de matéria seca das quatro espécies diminuiu com o aumento da dose de metais aplicada ao solo. Esse efeito foi marcante para o girassol, a mamoneira e a pimenta (Tabela 1), constatando-se que essas espécies foram sensíveis às doses de metais usadas no experimento. Por outro lado, quando foi aplicado EDTA no solo, houve diminuição na produção de matéria seca somente nas culturas da mamoneira e girassol, na dose mais elevada do metal. A produção de matéria seca das culturas de pimenta e tabaco não foi influenciada pelo aumento da dose de metais aplicada ao solo, quando este também recebeu EDTA (Tabela 1). Considerando somente a produção de massa seca da parte aérea, a cultura do tabaco foi a mais tolerante à aplicação dos metais ao solo (Tabela 1).

\subsection{Teores totais de metais pesados no solo e de Cd e Zn na parte aérea das culturas}

As concentrações totais de $\mathrm{Cu}, \mathrm{Ni}$ e $\mathrm{Pb}$ no solo foram respectivamente, em $\mathrm{mg} \mathrm{kg}^{-1}$ de: 7,0, 8,0 e 5,0 na dose zero de metais; e de 30, 14 e 10,0 na dose mais elevada. Esses valores concordam com os valores de referência de qualidade do solo de 35,13 e $17 \mathrm{mg} \mathrm{kg}^{-1}$ para o $\mathrm{Cu}, \mathrm{Ni}$ e $\mathrm{Pb}$, sugeridos pela CетеSв (2007) e estão dentro da faixa do teor normal observado nos solos do Estado de São Paulo, segundo CANCEla (2002). 
Comparando os teores de $\mathrm{Zn}\left(76 \mathrm{mg} \mathrm{kg}^{-1}\right)$ e Cd $\left(4 \mathrm{mg} \mathrm{kg}^{-1}\right)$ obtidos no presente trabalho com os valores orientadores sugeridos pela CETESB (2007), verifica- se que o teor de Zn estava um pouco acima daquele de referência de qualidade do solo $\left(60 \mathrm{mg} \mathrm{kg}^{-1}\right)$ e o $\mathrm{Cd}$, acima do valor de intervenção agrícola $\left(3 \mathrm{mg} \mathrm{kg}^{-1}\right)$.

Tabela 1. Efeito da adição ao solo de metais pesados e de EDTA na produção de matéria seca da parte aérea da mamoneira, girassol, pimenta e tabaco

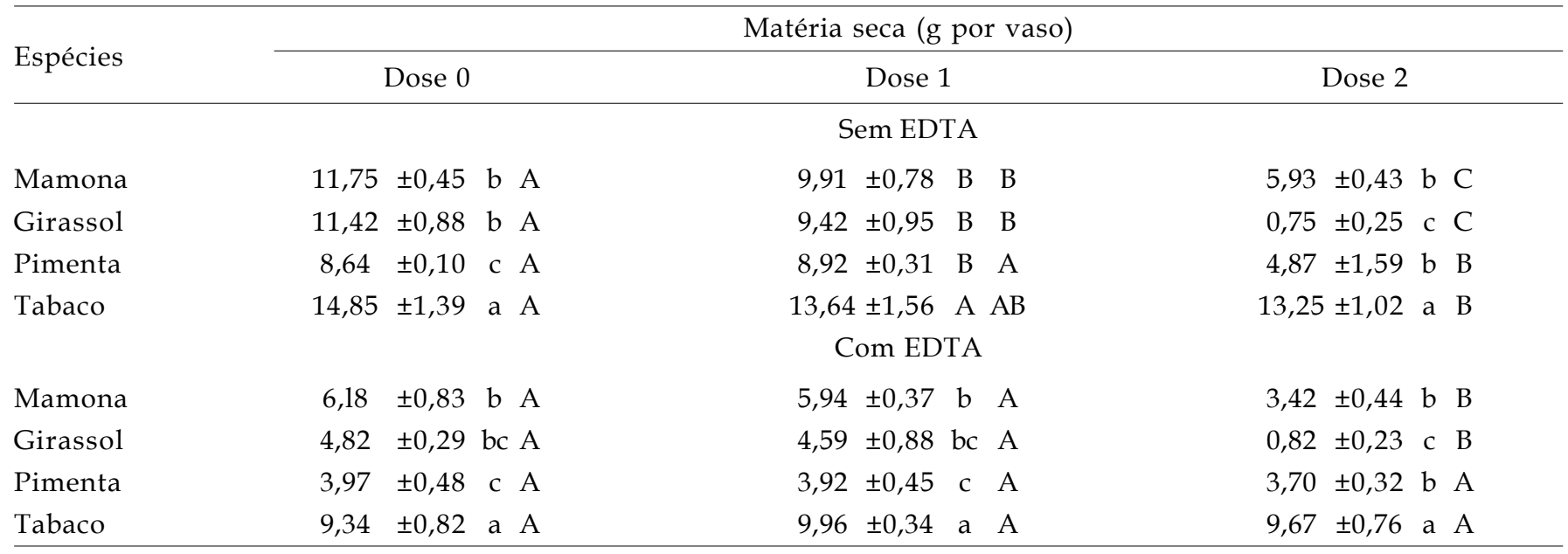

Médias de tratamentos seguidas pela mesma letra não diferem entre si pelo Teste de Tukey a 5\%; as letras minúsculas na mesma coluna comparam as espécies na ausência e presença de EDTA, e as maiúsculas na mesma linha, comparam as doses de metais.

A concentração de $\mathrm{Cd}$ na parte aérea das plantas variou, em $\mathrm{mg} \mathrm{kg}^{-1}$, de: 0,11 a 3,05 (mamoneiro); 0,28 a 9,26 (girassol); 0,35 a 4,23 (pimenta); e 0,60 a 13,30 (tabaco) (Tabela 2). O tabaco foi a espécie vegetal com maior concentração de $\mathrm{Cd}$. Para algumas situações, os teores desse metal podem ser considerados tóxicos, de acordo com KABATIAPendias e Pendias (1992), que sugerem o valor de 5,0 $\mathrm{mg} \mathrm{kg}^{-1}$ e com BERGMANN (1992) que sugere 4,0 mg $\mathrm{kg}^{-1}$, como valor tóxico para as plantas. FELIx et al. (1999) verificaram valores de $12,3 \mathrm{mg} \mathrm{kg}^{-1}$ de Cd na parte aérea de $N$. tabacum, crescido em solo contaminado com $6,6 \mathrm{mg} \mathrm{kg}^{-1}$ de Cd. Entretanto, esses autores verificaram que em espécies como Thlaspi caerulescens, Zea mays e Brassica juncea ocorreram variações nas concentrações de $\mathrm{Cd}$ similares àquelas verificadas neste experimento.

Comparando-se o efeito do EDTA, observouse que este provocou aumento na concentração de Cd na parte aérea da mamoneira e girassol e não teve influência na pimenta e no tabaco (Tabela 2). Aumentos de 1,5 a 5,3 vezes na absorção de $\mathrm{Cd}$ por plantas de Larix sibirica foram verificados por AvtuKHOVICH (2003), devido à aplicação de 0,372 mg. $\mathrm{kg}^{-1}$ de EDTA em solo que recebeu $10 \mathrm{mg} \mathrm{kg}^{-1}$ de Cd. Pereira (2005) verificou que a aplicação de 0,5 g. $\mathrm{kg}^{-1}$ de EDTA em um Latossolo do Estado de São Paulo foi eficiente em aumentar a concentração de $\mathrm{Pb}$ na parte aérea de plantas de feijão de porco e de girassol, não o sendo para as plantas de milho.
Embora a concentração de Cd na parte aérea das plantas tenha atingido níveis elevados, de acordo com o sugerido por BERGMANN (1992), nenhuma espécie testada foi considerada hiperacumuladora de $\mathrm{Cd}$, uma vez que o acúmulo desse elemento na parte aérea das plantas foi muito baixo (Tabela 2).

A concentração de $\mathrm{Zn}$ na parte aérea das plantas (Tabela 3) variou, em $\mathrm{mg} \mathrm{kg}^{-1}$, de: 51 a 1.188 (mamona), 50 a 1.800 (girassol), 32 a 564 (pimenta) e 52 a 899 (tabaco) (Tabela 3). Kabata-Pendias e Pendias (1992) sugerem valores de 100 a $400 \mathrm{mg} \mathrm{kg}^{-1} \mathrm{e}$ Bergmann (1992) de 300 a $500 \mathrm{mg} \cdot \mathrm{kg}^{-1}$, como tóxicos. Percebeu-se que, com exceção do tratamentotestemunha, a concentração de Zn na parte aérea de todas as espécies estava alta e, para a maioria dos casos, tóxica de acordo com os valores sugeridos pelos autores citados anteriormente. De maneira contrária ao verificado para o $\mathrm{Cd}$, a mamona e o girassol foram as espécies que tiveram as maiores concentrações de Zn (Tabela 3).

Comparando o efeito do EDTA, observou-se que este não afetou significativamente a concentração de Zn na parte aérea de todas as espécies testadas (Tabela 3). Provavelmente, a eficiência do EDTA em aumentar a absorção de $\mathrm{Zn}$ pelas diferentes espécies foi reduzida pela presença de outros elementos que possuem afinidade pelo complexante tais como $\mathrm{Fe}, \mathrm{Cu}, \mathrm{Al}$ e $\mathrm{Cd}$. Conforme BACCAN et al. (2001), dentre os metais presentes no solo, observa-se no Fe e no Al grande afinidade pelo EDTA, com elevadas constantes de formação. 
Tabela 2. Efeito da adição ao solo de metais pesados e de EDTA sobre a concentração e o acúmulo de Cd na parte aérea da mamoneira, girassol, pimenta e tabaco

\begin{tabular}{|c|c|c|c|c|}
\hline \multirow{2}{*}{ Espécies } & \multicolumn{4}{|c|}{ Cádmio } \\
\hline & \multicolumn{2}{|r|}{ Dose 0} & Dose 1 & Dose 2 \\
\hline & \multicolumn{4}{|c|}{ Concentração na parte aérea $\left(\mathrm{mg} \mathrm{kg}^{-1}\right)$} \\
\hline & \multicolumn{4}{|c|}{ Sem EDTA } \\
\hline Mamona & 0,11 & $\pm 0,02$ a $\mathrm{B}$ & $0,62 \pm 0,03 \quad$ с $\mathrm{AB}$ & $2,28 \pm 0,16$ c $\mathrm{A}$ \\
\hline Girassol & 0,28 & $\pm 0,00$ a $C$ & $4,60 \pm 0,86$ b $B$ & $9,26 \pm 3,76$ b A \\
\hline Pimenta & 0,43 & $\pm 0,22$ a $\mathrm{B}$ & $2,55 \pm 0,49$ bc $A$ & $4,23 \pm 0,42$ c $\mathrm{A}$ \\
\hline \multirow[t]{2}{*}{ Tabaco } & 0,61 & $\pm 0,23$ a $C$ & $10,41 \pm 1,18$ a $B$ & $12,30 \pm 1,89$ a $\mathrm{A}$ \\
\hline & \multicolumn{4}{|c|}{ Com EDTA } \\
\hline Mamona & 0,29 & $\pm 0,03$ a $\mathrm{B}$ & $1,13 \pm 0,16 \quad b \quad B$ & $3,05 \pm 0,92 \mathrm{~b} \quad \mathrm{~A}$ \\
\hline Girassol & 0,54 & $\pm 0,05$ a $C$ & $8,07 \pm 0,84$ a $A$ & $3,63 \pm 0,31 \quad b \quad B$ \\
\hline Pimenta & 0,35 & $\pm 0,05$ a $B$ & $2,78 \pm 0,09$ b $A$ & $4,00 \pm 0,64 \mathrm{~b} \quad \mathrm{~A}$ \\
\hline \multirow[t]{3}{*}{ Tabaco } & 0,60 & $\pm 0,09$ a $\mathrm{B}$ & $8,79 \pm 2,27$ a $\mathrm{A}$ & $9,15 \pm 1,36$ a $\mathrm{A}$ \\
\hline & \multicolumn{4}{|c|}{ Acúmulo na parte aérea (mg por vaso) } \\
\hline & \multicolumn{4}{|c|}{ Sem EDTA } \\
\hline Mamona & 0,001 & $\pm 0,00$ a $\mathrm{A}$ & $0,006 \pm 0,00$ d $A$ & $0,014 \pm 0,001 \mathrm{~b} \quad \mathrm{~A}$ \\
\hline Girassol & 0,003 & $\pm 0,00$ a $\mathrm{B}$ & $0,043 \pm 0,009 \mathrm{~b} \quad \mathrm{~A}$ & $0,007 \pm 0,003$ b $\quad$ B \\
\hline Pimenta & 0,004 & $\pm 0,002$ a $\mathrm{B}$ & $0,023 \pm 0,004$ с $\mathrm{A}$ & $0,021 \pm 0,008 \mathrm{~b} \quad \mathrm{~A}$ \\
\hline \multirow[t]{2}{*}{ Tabaco } & 0,009 & $\pm 0,003$ a $C$ & $0,141 \pm 0,017$ a $\mathrm{B}$ & $0,162 \pm 0,019$ a $\mathrm{A}$ \\
\hline & \multicolumn{4}{|c|}{ Com EDTA } \\
\hline Mamona & 0,002 & \pm 0 a $\mathrm{A}$ & $0,007 \pm 0,001$ с $A$ & $0,011 \pm 0,004 \mathrm{~b} \quad \mathrm{~A}$ \\
\hline Girassol & 0,003 & $\pm 0 \quad$ a $\quad \mathrm{B}$ & $0,037 \pm 0,009 \mathrm{~b} \quad \mathrm{~A}$ & $0,003 \pm 0,001 \mathrm{~b} \quad \mathrm{~B}$ \\
\hline Pimenta & 0,001 & $\pm 0 \quad$ a $\mathrm{A}$ & $0,011 \pm 0,001$ с $\mathrm{A}$ & $0,015 \pm 0,003 \mathrm{~b} \quad \mathrm{~A}$ \\
\hline Tabaco & 0,006 & $\pm 0,001$ a $\mathrm{B}$ & $0,088 \pm 0,023$ a $A$ & $0,088 \pm 0,010$ a $\mathrm{A}$ \\
\hline
\end{tabular}

Médias de tratamentos seguidas pela mesma letra não diferem entre si pelo Teste de Tukey a 5\%; as letras minúsculas na mesma coluna comparam as espécies na ausência e presença de EDTA, e as maiúsculas na mesma linha comparam as doses de metais.

O aumento da concentração de $\mathrm{Zn}$ na parte aérea das quatro espécies, independentemente da adição ou não do complexante, foi proporcional ao aumento da dose de metais (Tabela 3). Por exemplo, o teor de $\mathrm{Zn}$ na parte aérea do tabaco crescido na ausência de EDTA foi de 435 e $899 \mathrm{mg} \mathrm{kg}^{-1}$ quando

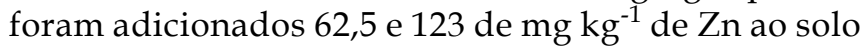
respectivamente.

No que se refere à quantidade de $\mathrm{Zn}$ acumulada na parte aérea das plantas, os efeitos da adição de EDTA e dos metais pesados foram muito parecidos àqueles observados para a concentração de Zn na parte aérea da mamona, girassol, pimenta e tabaco (Tabela 3). Considerando essa característica, nenhuma planta foi considerada hiperacumuladora de $\mathrm{Zn}$.

\subsection{Balanço de massa}

O balanço de massa permite avaliar a eficiência de cada uma das espécies em extrair o Cd e o $\mathrm{Zn}$ do solo, na ausência e na presença do complexante EDTA. Para tanto, estimaram-se as quantidades de metais pesados a serem removidos do solo, de acordo com a adição de cada elemento (Tabela 4 ).

A eficiência das espécies em remediar uma área contaminada com $\mathrm{Cd}$ foi muito diferente levando-se em consideração o número de cultivos necessários para cada espécie (Tabela 5). Dentre as espécies, o tabaco foi a mais eficiente sendo necessários 15 cultivos para remover o $\mathrm{Cd}$ do solo (Tabela 5). No tratamento correspondente à aplicação da dose 2 de metal e, na ausência de EDTA, a cultura do tabaco retirou $6,53 \%$ do $\mathrm{Cd}$ excedente do solo. CARneiro et al. (2001), desenvolvendo experimentos de implantação de culturas em solo contaminado por metais pesados, obtiveram cerca de $9 \%$ de extração de Cd por Brassica juncea, o que foi considerado pelos autores como um indicativo de alta capacidade de fitoextração. 
A adição do complexante EDTA também afetou o número de cultivos necessários para a remoção do Cd do solo (Tabela 5).

Com exceção da mamoneira, será preciso menor número de cultivos para todas as culturas se não houver a aplicação de EDTA (Tabela 5).
De maneira semelhante ao observado para o $\mathrm{Cd}$, será necessário menor número de cultivos de todas as plantas testadas para retirada do $\mathrm{Zn}$ do solo se não aplicar EDTA (Tabela 6). As culturas do tabaco e girassol se destacaram como as plantas mais eficientes (Tabela 6).

Tabela 3. Efeito da adição de metais pesados e de EDTA sobre a concentração e acúmulo de Zn na parte aérea da mamoneira, girassol, pimenta e tabaco

\begin{tabular}{lc}
\hline Espécies & Dose 0 \\
\cline { 2 - 2 } & \\
& \\
Mamona & $51,05 \pm 4,23$ a C \\
Girassol & $50,53 \pm 2,19$ a C \\
Pimenta & $32,48 \pm 8,29$ a B \\
Tabaco & $52,05 \pm 19,74$ a C \\
& \\
Mamona & $73,28 \pm 9,01$ a C \\
Girassol & $94,55 \pm 4,17$ a C \\
Pimenta & $36,13 \pm 2,39$ a C \\
Tabaco & $54,48 \pm 9,97$ a C
\end{tabular}

Concentração na parte aérea $\left(\mathrm{mg} . \mathrm{kg}^{-1}\right)$

Sem EDTA

$\begin{array}{ccc}308,55 \pm 18,57 & \text { b } & \text { B } \\ 716,35 \pm 29,89 & \text { a } & \text { B } \\ 142,78 \pm 9,73 & \text { c } & \text { B } \\ 435,08 \pm 67,43 & \text { b } & \text { B } \\ \text { Com EDTA } & \\ 469,95 \pm 60,73 & \text { b } & \text { B } \\ 1335,08 \pm 140,14 & \text { a } & \text { B } \\ 180,78 \pm 14,27 & \text { c } & \text { B } \\ 451,35 \pm 99,03 & \text { b } & \text { B }\end{array}$

$717,28 \pm 44,25$ с A

$1800,53 \pm 233,11$ a $\mathrm{A}$

$564,88 \pm 55,00 \mathrm{~d} \mathrm{~A}$

$899,28 \pm 139,64$ b A

$1188,40 \pm 65,18$ b A

$1785,20 \pm 128,32$ a $\mathrm{A}$

$486,85 \pm 47,97$ d A

$822,10 \pm 104,77$ c A

Acúmulo na parte aérea (mg por vaso)

Sem EDTA

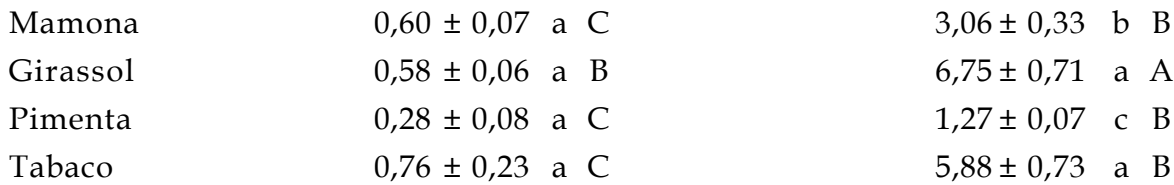

$4,25 \pm 0,33$ b $A$

$1,32 \pm 0,38$ d B

$2,72 \pm 0,84$ с A

$11,84 \pm 1,41$ a $\mathrm{A}$

Com EDTA

$2,79 \pm 0,39$ с $B$

$6,03 \pm 0,66$ a $\mathrm{A}$

$0,71 \pm 0,11$ d B

$4,06 \pm 0,51$ b A

$1,45 \pm 0,37$ с $B$

$1,79 \pm 0,03$ с A

$4,49 \pm 0,94$ b $B$

$7,89 \pm 0,45$ a $\mathrm{A}$

Médias de tratamentos seguidas pela mesma letra não diferem entre si pelo Teste de Tukey a 5\%; as letras minúsculas na mesma coluna comparam as espécies, e as maiúsculas na mesma linha comparam as doses de metais.

Tabela 4. Teores totais e quantidade a ser removida de Cd e $\mathrm{Zn}$ do solo contaminado

\begin{tabular}{|c|c|c|c|c|c|c|}
\hline \multicolumn{2}{|c|}{ Solo contaminado } & \multirow{3}{*}{$\begin{array}{c}\text { Solo não contaminado } \\
\mathrm{mg} \cdot \mathrm{kg}^{-1}\end{array}$} & \multicolumn{2}{|c|}{ A ser removido } & \multirow{2}{*}{\multicolumn{2}{|c|}{$\begin{array}{l}\text { Quantidade a ser } \\
\text { removida do vaso } \\
\text { mg por vaso }\end{array}$}} \\
\hline & & & & & & \\
\hline Dose 1 & Dose 2 & & $\begin{array}{l}\text { Dose } 1 \\
\text { Cádmio }\end{array}$ & Dose 2 & Dose 1 & Dose 2 \\
\hline 3,70 & 4,03 & 2,98 & $\begin{array}{r}0,72 \\
\text { Zinco }\end{array}$ & 1,05 & 2,16 & 3,15 \\
\hline 47,45 & 76,54 & 16,51 & 30,94 & 60,03 & 92,82 & 180,09 \\
\hline
\end{tabular}

Média referente às amostras de terra obtidas logo após a adição dos metais e antes do cultivo da mamoneira, girasol, pimenta e tabaco. Média referente às amostras de terra obtidas nos tratamentos que não receberam metais pesados. 
Tabela 5. Eficiência das culturas em remover o $\mathrm{Cd}$ do solo e o número de cultivos necessários para remediar o solo contaminado

\begin{tabular}{|c|c|c|c|c|c|}
\hline \multirow[b]{2}{*}{ Cultura } & \multirow[b]{2}{*}{ EDTA } & \multirow[b]{2}{*}{$\begin{array}{l}\text { Quantidade acumulada } \\
\text { na parte aérea }\end{array}$} & \multirow[b]{2}{*}{$\begin{array}{l}\text { Eficiência } \\
\text { da cultura }\end{array}$} & \multicolumn{2}{|c|}{ Remediação total } \\
\hline & & & & $\begin{array}{l}\text { Matéria seca } \\
\text { produzida }\end{array}$ & $\begin{array}{c}\text { Número } \\
\text { de cultivos }\end{array}$ \\
\hline & & mg por vaso & $\%$ & g por vaso & \\
\hline \multirow[t]{2}{*}{ Mamona } & Sem & 0,006 & 0,28 & 3567,60 & 360 \\
\hline & Com & 0,007 & 0,33 & 1832,91 & 309 \\
\hline \multirow[t]{2}{*}{ Girassol } & Sem & 0,043 & 1,99 & 473,17 & 50 \\
\hline & Com & 0,037 & 1,71 & 267,97 & 59 \\
\hline \multirow[t]{2}{*}{ Pimenta } & Sem & 0,023 & 1,07 & 837,68 & 94 \\
\hline & Com & 0,011 & 0,51 & 769,73 & 196 \\
\hline \multirow[t]{2}{*}{ Tabaco } & Sem & 0,141 & 6,53 & 208,97 & 15 \\
\hline & Com & 0,088 & 4,08 & 244,52 & 25 \\
\hline \multirow[t]{2}{*}{ Mamona } & Sem & 0,014 & 0,45 & 1334,25 & 225 \\
\hline & Com & 0,011 & 0,35 & 979,35 & 286 \\
\hline \multirow[t]{2}{*}{ Girassol } & Sem & 0,007 & 0,22 & 337,50 & 450 \\
\hline & Com & 0,003 & 0,10 & 861,00 & 1050 \\
\hline \multirow[t]{2}{*}{ Pimenta } & Sem & 0,021 & 0,67 & 730,50 & 150 \\
\hline & Com & 0,015 & 0,48 & 777,00 & 210 \\
\hline \multirow[t]{2}{*}{ Tabaco } & Sem & 0,162 & 5,14 & 257,58 & 19 \\
\hline & Com & 0,088 & 2,79 & 346,19 & 36 \\
\hline
\end{tabular}

Tabela 6. Eficiência das culturas em remover o $\mathrm{Zn}$ do solo e o número de cultivos necessários para remediar o solo contaminado

\begin{tabular}{|c|c|c|c|c|c|}
\hline \multirow[b]{2}{*}{ Cultura } & \multirow[b]{2}{*}{ EDTA } & \multirow{2}{*}{$\begin{array}{l}\text { Quantidade } \\
\text { absorvida }\end{array}$} & \multirow{2}{*}{$\begin{array}{l}\text { Eficiência } \\
\text { da cultura }\end{array}$} & \multicolumn{2}{|c|}{ Remediação Total } \\
\hline & & & & $\begin{array}{c}\text { Matéria seca } \\
\text { produzida }\end{array}$ & $\begin{array}{c}\text { Número } \\
\text { de cultivos }\end{array}$ \\
\hline & & mg por vaso & $\%$ & g por vaso & \\
\hline \multirow{2}{*}{ Mamona } & Sem & 3,06 & 3,33 & 300,27 & 30 \\
\hline & Com & 2,79 & 3,01 & 197,80 & 33 \\
\hline \multirow[t]{2}{*}{ Girassol } & Sem & 6,75 & 7,27 & 129,53 & 14 \\
\hline & Com & 6,03 & 6,5 & 70,64 & 15 \\
\hline \multirow[t]{2}{*}{ Pimenta } & Sem & 1,27 & 1,37 & 651,96 & 73 \\
\hline & Com & 0,71 & 0,77 & 512,34 & 131 \\
\hline \multirow[t]{3}{*}{ Tabaco } & Sem & 5,88 & 6,33 & 215,38 & 16 \\
\hline & Com & 4,49 & 4,84 & 205,87 & 21 \\
\hline & & & - Dose 2 & & \\
\hline \multirow[t]{2}{*}{ Mamona } & Sem & 4,25 & 2,36 & 251,26 & 42 \\
\hline & Com & 4,06 & 2,26 & 151,71 & 44 \\
\hline \multirow[t]{2}{*}{ Girassol } & Sem & 1,32 & 0,73 & 102,32 & 136 \\
\hline & Com & 1,45 & 0,81 & 101,85 & 124 \\
\hline \multirow[t]{2}{*}{ Pimenta } & Sem & 2,72 & 1,51 & 322,44 & 66 \\
\hline & Com & 1,79 & 0,99 & 372,26 & 101 \\
\hline \multirow[t]{2}{*}{ Tabaco } & Sem & 11,84 & 6,58 & 201,53 & 15 \\
\hline & Com & 7,89 & 4,38 & 220,77 & 23 \\
\hline
\end{tabular}




\section{CONCLUSÕES}

1. A planta mais eficiente em extrair o $\mathrm{Cd}$ e o $\mathrm{Zn}$ do solo enriquecido com metais foi o tabaco (N. tabacum).

2. A aplicação de $1 \mathrm{mmol} \mathrm{kg}^{-1}$ de EDTA no solo não contribuiu para melhorar a eficiência das plantas em relação à extração dos metais pesados em solos.

\section{REFERÊNCIAS}

ABREU, M.F.;ABREU, C.A.;ANDRADE, J.C. Determinação de fósforo, potássio, cálcio, magnésio, enxofre, cobre, ferro, manganês, zinco, níquel, cádmio, cromo, e chumbo em ácido nítrico usando métodos da US-EPA. In: RAIJ, B.;ANDRADE, J.C.;CANTARELLA, H.;QUAGGIO, J.A. (Ed.). Análise química para avaliação da fertilidade de solos tropicais. Campinas: Instituto Agronômico, 2001. p.251-261.

ACCIOLY, A.M.A.;SIQUEIRA, J.O. Contaminação química e biorremediação do solo. In: NOVAIS, R.F.;ALVAREZ, V.H.;SCHAEFER, C.E.G.R. (Ed.).Tópicos em Ciência do Solo. Viçosa: Sociedade Brasileira de Ciência doSolo, 2000. p.299-352.

ASHMORE, M. Plants and pollution. In: CRAWLEY, M.J. (Ed.) Plant Ecology. 2 ed. Oxford:Blackwell Science, 2000. p.568-581.

AVTUKHOVICH, I. Study of distribution of Cd in soil fractions and increase in the efficiency of phytoextraction using EDTA. In: INTERNATIONAL CONFERENCE ON THE BIOGEOCHEMISTRY OF TRACE ELEMENTS, 7., 2003 UpsalaSweden: SLU Service, 2003. p. 126-127.

BATAGLIA, O.C.;FURLANI, A.M.C.;TEIXEIRA, J.P.F.;FURLANI, P.R.;GALLO, J.R. Métodos de análise química de plantas. Campinas: Instituto Agronômico, 1983.48p. (Boletim técnico n.78)

BACCAN, N.; ANDRADE, J.C.; GODINHO, O.E.S; BARONE, J.S. Titulações complexométricas. In: Química Analítica Quantitativa Elementar. Campinas: Edgad Blücher, 2001. p.133-134.

BERGMANN, W. Nutrition disorders of plants-development, visual and analytical diagnosis. Stuttgart: Gustaf Fischer Verlag Jena, 1992. 741p.

CARNEIRO, M.A.C.; SIQUEIRA, J.O.; MOREIRA, F.M.C. Estabelecimento de plantas herbáceas em solo com contaminação de metais pesados e inoculação de fungos micorrízicos arbusculares. Pesquisa Agropecuária Brasileira, v.36, n.12, p.1443-1452, 2001.

CANCELA, R. Contenido de macro-,micronutrientes, metales pesados y otros elementos en suelos naturales de São Paulo (Brasil) y Galicia (España). 2002. 574f. Tesis (Doctoral). Universidad de La Coruña, Spain, 2002.

CETESB. COMPANHIA DE TECNOLOGIA DESANEAMENTO AMBIENTAL. Relação de áreas contaminadas no Estado de São Paulo. Disponível em: http:/www.cetesb.sp.gov.br. Acesso em 29 de janeiro de 2007.

CHEN,H.; CUTRIGHT, T. EDTA and HEDTA effects on Cd, Cr and Ni uptake by Helianthus annuus. Chemosphere, v.45, p.21-28, 2001.

ERNEST, W.H.O. Bioavailability of heavy metals and decontamination of soils by plants. Applied Geochemistry, v.11, p.163-167, 1996.
FELIX, H.R.; KAYSER, A.; SCHULIN, R. Phytoremediation field trials in the years 1993-1998. In: INTERNATIONAL CONFERENCE ON THE BIOGEOCHEMESTRY OF TRACE ELEMENTS, 5., 1999, Vienna - Austria: s.n., 1999. p.8-9.

GARBISU, C.; ALKORTA, I. Phytoextraction: a cost effective plant-based technology for the removal of metals from the environment. Bioresource Technology, v. 77, p. 229 -236, 2001.

HUANG, J.W.; CHEN, J.; BERTI, W.R.; CUNNINGHAM, S.D. Phytoremediation of lead-contaminated soils: role of synthetic chelates in lead phytoextraction. Environmental Science Technology, v.31, p.800-805, 1997.

KABATA-PENDIAS, A.; PENDIAS, H. Trace elements in soils and plants. 2.ed. Boca Raton: CRC Press, 1992. 413p.

KHAN, A.G.; KUEK, C.; CHAUDHRY, T.M.; KHOO, C.S; HAYES, W.J. Role of plants, mycorrhizae and phytochelators in heavy metal contaminated land remediation. Chemosphere, v.41, p.197-207, 2000.

MARQUES, T.C.L.L.S.M.; MOREIRA, A.M.S.; SIQUEIRA, J.O. Crescimento e teor de metais de mudas de espécies arbóreas cultivadas em solo contaminado com metais pesados. Pesquisa Agropecuária Brasileira, Brasília, v.35, n.1, p.121-132, 2000.

MOHR, H.; SCHOPFER, P. Plant physiology. Berlim: SpringerVerlag, 1995. 629p.

PEREIRA, B.F.F. Potencial fitorremediador das culturas de feijão de porco, girassol e milho cultivadas em Latossolo Vermelho contaminado com chumbo. 2005. 68 f. Dissertação (Mestrado) - Instituto Agronômico, Campinas, 2005.

RASKIN, I.; KUMAR, PBA. N.; DUSHENKOV, S.; SALT, D.E. Bioconcentration of heavy metals by plants. Current opinions in Biotechnology, v.5, p.285-290, 1994.

ROUTH, G.R.; SAMANTARAY, S.; DAS, P.; ROSTLINNA, V. Differential lead tolerance of rice and black gram in hydroponic culture. Rostlinna Vyroba, v.47: n.12, p. 541-548, 2001.

SHEN, Z.G.;LI, X.D.; WANG, C.C.; CHEN, H.M.; CHUA, H. Lead phytoextraction from contaminated soil with high-biomass plant species. Journal of Environmental Quality, v.31, p.1893-1900, 2002.

TANDY, S.; BOSSART, K.; MUELLER, R.; RITSCHEL, J.; HAUSER, L.; SCHULIN, R.; NOWACK, B. Extraction of heavy metals from soils using biodegradable chelating agents. Environmental Science Technology, v.38, p.937-944, 2004.

UNITEDSTATES ENVIRONMENTAL PROTECTION AGENCY (US-EPA). Introduction to phytoremediation: EPA/600/R-99/ 107. Cincinatti: National Risk Management Research Laboratory, 2000.

UNITEDSTATES ENVIRONMENTAL PROTECTION AGENCY (US-EPA). The Clean Green - Phytoremediation: Produced by U.S. EPA Environmental Response Team. Disponível na internet: <http://www.clu-in.org/studio/video.cfm>. Acesso em 1. ${ }^{\circ}$ de outubro de 2004.

ZONTA, E.P.; MACHADO, A.A.; SILVEIRA, P. Sistema de análise estatística para microcomputadores (Sanest). Pelotas: Universidade Federal de Pelotas, 1984. 75p. 